\title{
Impact of Laparoscopic Sleeve Gastrectomy on Gastro Esophageal Reflux Disease
}

\author{
Ramy Mikhael Nageeb, MD; Wafi Fouad, MD; Dina Hany, MD \\ Department of General Surgery, Ain Shams University, Egypt
}

Introduction: Although laparoscopic sleeve gastrectomy (LSG) is an effective treatment for morbid obesity, the effects of LSG on gastroesophageal reflux disease (GERD) are controversial. Although improvement of GERD symptoms following LSG has been reported in some studies, others have shown a 9-34.6\% increase in the prevalence of GERD symptoms during the first year following LSG.

Aim: This study was aiming to evaluate the changes of GERD symptoms and erosive esophagitis (EE) in morbidly obese patients who underwent LSG.

Patients and methods: Pre-and post-operative details of forty morbidly obese patients (body mass index (BMI) more than $40 \mathrm{~kg} / \mathrm{m}^{2}$ or $35 \mathrm{~kg} / \mathrm{m}^{2}$ with other comorbidities) with and without GERD who underwent laparoscopic sleeve gastrectomy and were followed up in Ain Shams University hospitals between September 2014 and May 2016 were recorded.

Results: After surgery, significant decreases were reported in mean body mass index $(44.3 \pm 3.8$ vs. $\left.36.5 \pm 5.3 \mathrm{~kg} / \mathrm{m}^{2}\right)$. Conversely, an insignificant increase was observed in the prevalence and severity of GERD symptoms and EE after LSG. 1 patient (20\%) developed denovo symptomatic GERD within the 6 months follow-up.

Conclusion: Although LSG can achieve significant weight loss and improvement of comorbidities in severely obese patients, the prevalence and severity of GERD symptoms and EE increase after the operation mandates that it should be carefully investigated pre-operatively and other bariatric surgery options should be considered.

Key words: LSG, GERD, BMI, EE.

\section{Introduction}

The incidence of morbid obesity has been steadily increasing worldwide and is now commonly referred to as an epidemic. Morbid obesity has been associated with multiple adverse health effects, including mortality and morbidity from heart disease, diabetes mellitus, sleep apnea, osteoarthritis, and hypertension. ${ }^{1,2}$

An additional common weight-related comorbidity is gastroesophageal reflux disease (GERD). They are often associated, especially because obesity increases the intra-abdominal pressure, generating the forces necessary to cause the reflux. ${ }^{3,4}$

Bariatric surgery, when combined with lifestyle and medical interventions, is a common and successful treatment modality in the obese patient. Laparoscopic sleeve gastrectomy (LSG) is one such procedure that has increased in popularity as a definitive bariatric operation. ${ }^{5}$ Laparoscopic sleeve gastrectomy has been proven to be an effective weight loss procedure and has gained wide acceptance as stand- alone surgical option for the treatment of morbid obesity. It is technically easier and is associated with lower postoperative morbidity and mortality rates. Nevertheless, this procedure carries one potential drawback namely gastroesophageal reflux disease (GERD). ${ }^{6}$

Data concerning the effects of LSG on esophageal function and GERD are limited and controversial. Some small studies have suggested that anatomical changes associated with LSG may exacerbate GERD symptoms or induce GERD in previously asymptomatic patients. Additionally, the recent literature can be divided into two categories: those that support an increase in GERD prevalence after LSG and those that demonstrate a decrease in GERD prevalence after LSG. ${ }^{7}$ However, these series have raised significant concern and debate about the effect of LSG on GERD and whether GERD may even be a relative contraindication to LSG.

\section{Patients and methods}

The study population consisted of forty consecutive morbidly obese patients eligible for LSG performed 
and evaluated in Ain Shams University hospitals between September 2014 and May 2016. Patients with a body mass index (BMI) more than $40 \mathrm{~kg} / \mathrm{m}^{2}$ (or $35 \mathrm{~kg} / \mathrm{m}^{2}$ with other comorbidities) who were unable to reduce their BMI via dieting, behavior modification, or pharmacologic therapy were considered for bariatric surgery, patients with GERD (clinically and endoscopically proven) were also included in our study. Exclusion criteria were the presence of hiatal hernia, heavy sweet eaters and previous gastric surgery. A detailed explanation was made to the patients about our study and the duration of follow-up and signed informed consent was obtained.

Preoperative workup included detailed history, clinical examination, upper GIT endoscopy and barium swallow. BMI was calculated as the weight in kilograms divided by the square of height in meters $\left(\mathrm{kg} / \mathrm{m}^{2}\right)$. All patients were surveyed about the presence of typical (heartburn, regurgitation and dysphagia) and atypical (cough, chest pain) GERD symptoms. The severity of symptoms was scored as 0 (no symptoms), 1 (mild, easily controlled with antacids), 2 (moderate, controlled by proton pump inhibitors), 3 (severely affecting quality of life). The frequency of symptoms was scored as follows: 0 : absent; 1: once/month; 2: once/week; 3: two to four times/week; 4 : daily.

All patients underwent endoscopy for preoperative evaluation. Diagnosis and classification of esophagitis was based on the Los Angeles (LA) classification, according to this system, esophagitis was classified as grade A (one or more mucosal breaks below $5 \mathrm{~mm}$ in length that do not extend between the tops of two mucosal folds), grade B (one or more mucosal breaks $5 \mathrm{~mm}$ in length that do not extend between the tops of two mucosal folds), grade $\mathrm{C}$ (one or more continuous mucosal breaks between the tops of two or more mucosal folds involving less than $75 \%$ of the esophageal circumference), and grade $\mathrm{D}$ (one or more mucosal breaks that involve at least $75 \%$ of the esophageal circumference).

Barium Swallow was performed to detect and exclude patients with short esophagus and hiatal hernia.

\section{Surgical Technique:}

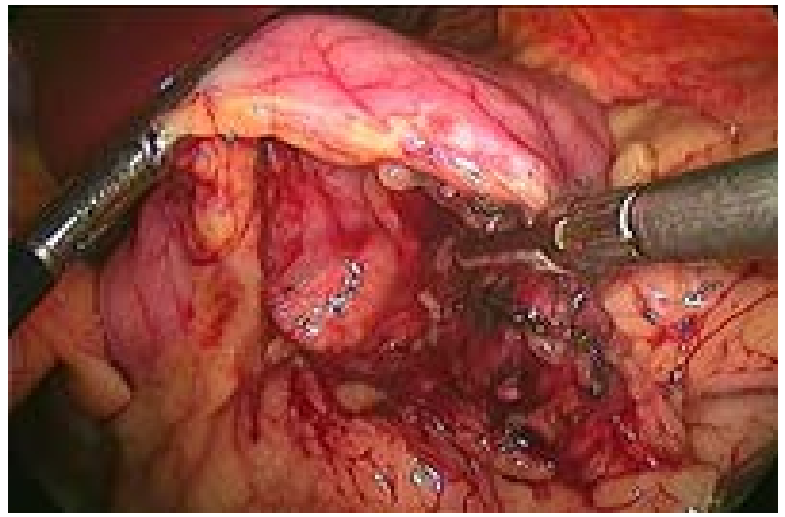

Fig 1: Division of the gastrocolic omentum.

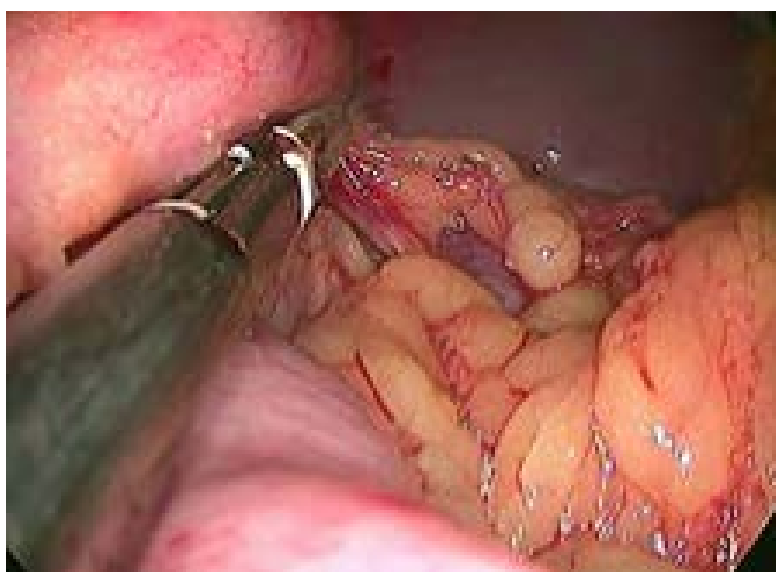

Fig 2: Division of short gastric vessels.

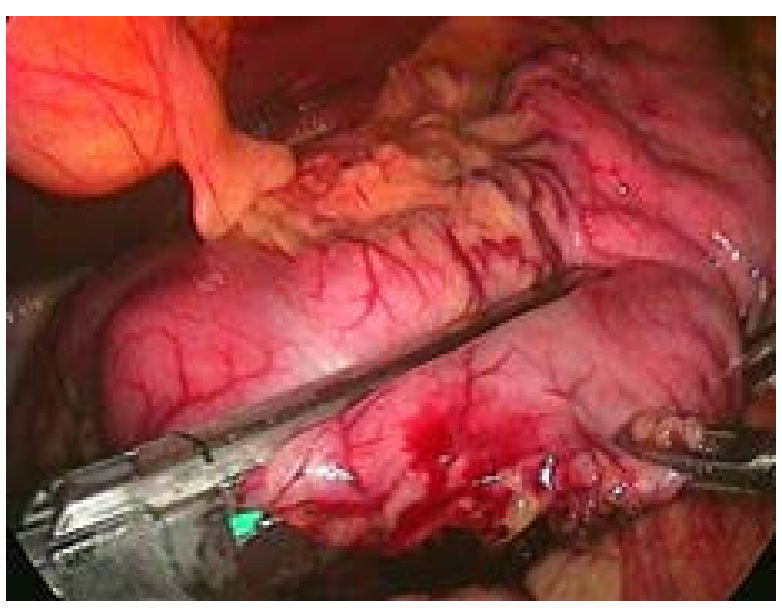

Fig 3: Creating gastric sleeve. 


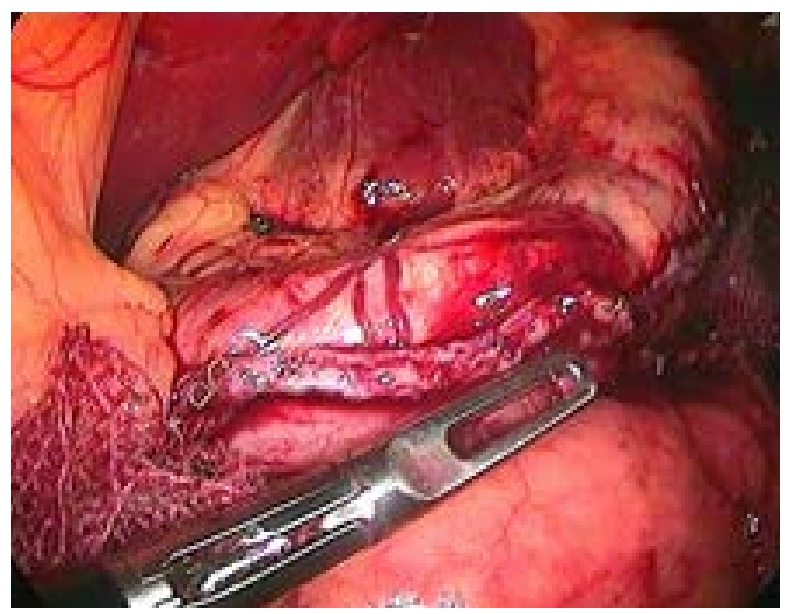

Fig 4: The final shape of the gastric sleeve.

Antiembolic precautions weretaken and appropriate preoperative antibiotics were administered. The patient was placed in the semi-lithotomy position in an anti-Trendelenburg position. The skin of the abdomen was prepped and draped in the usual sterile fashion. Pneumoperitoneum was induced using the Verress needle in the left upper quadrant. Once the pneumoperitoneum was completed at 14 $\mathrm{mmHg}$, a 12-mm trocar was placed in the midline supraumbilical position approximately $15 \mathrm{~cm}$ below the xiphoid. Four more trocars (two $12 \mathrm{~mm}$, and two $5 \mathrm{~mm}$ ) were then placed in right and left midclavicular lines, and left mid-axillary line. The liver was retracted using a self-retaining liver retractor via an epigastric (subxiphisternal) port.

The surgery started by the division of the gastrocolic omentum (Figure 1), starting 5-6 cm proximal to the pylorus using the Harmonic Scalpel and proceeding up to the angle of His. We ensured that there was at least $3 \mathrm{~cm}$ of intra-abdominal esophagus. The short gastric vessels were identified and divided (Figure 2). Dissection was performed up to the left crus of the esophageal hiatus, and all attachments were released to completely mobilize the fundus. The fundus and the body of the stomach were carefully mobilized by isolating posterior adhesions to the anterior capsule of the pancreas.

The gastric sleeve was created (Figures $\mathbf{3 , 4}$ ) using sequential firings of a $60-\mathrm{mm}$ linear stapling device. The staplers were applied alongside a 36Fr calibrating bougie positioned in the stomach against the lesser curve so as to avoid stenosis and to obtain a narrow gastric tube. We fired a $60-4.8-\mathrm{mm}$ staple cartridge initially, followed by three to five $60-3.5-\mathrm{mm}$ cartridges, carefully avoiding relative narrowing at the junction between the vertical and horizontal parts of the stomach, which usually occurred during the first, and occasionally the second firing of the stapler if it was inappropriately pressed against the bougie in an attempt to leave a small antrum. Narrowing was avoided by using an articulating stapler, slightly angled to the greater curvature, to create a wide angle at the junction between the horizontal antrum and the vertical body of the stomach. When firing the staplers, it was important not to overstretch the stomach to avoid narrowing by recoil of the stapler line.

The bougie was withdrawn, and a leak test was performed using intragastric methylene blue dye. The resected specimen was retrieved via the 12 $\mathrm{mm}$ port. A suction drain was placed in the lesser sac alongside the stable line, hemostasis was ensured, and port sites were closed.

The patient was discharged the following day after tolerating a liquid diet and receiving a complete set of instructions which included aspects of nutrition, medication, activity, appointments, and mental health. The patient was evaluated on the eighth postoperative day and then at 1, 3 and 6 months postoperatively. We recorded symptoms of GERD using the same survey as in the preoperative evaluation. Upper GIT endoscopy was indicated if the patient had difficulty tolerating solid food and on the sixth postoperative month in patients with symptoms of GERD (pre- or post-operatively).

\section{Statistical Analysis:}

Data were analyzed using Fisher's and chi-square test. All tests were considered significant if $(p \leq 0.05)$.

\section{Results}

\section{Patient demographics:}

From September 2014 to May 2016, a total of 40 morbidly obese patients underwent LSG in Ain Shams University hospitals. Of those forty patients, there were 32 (80\%) females and 8 (20\%) males, mean age was $42.6 \pm 10.2$ years and the BMI was $44.3 \pm 3.8 \mathrm{~kg} / \mathrm{m}^{2} .35(87.5 \%)$ patients had GERD symptoms preoperatively, $17(42.5 \%)$ patients had hypertension and 21 (52.5\%) patients had type 2 DM. Table 1 shows baseline characteristics of patients with and without GERD.

Table 1: Patients' characteristics with and without GERD. BMI: body mass index

\begin{tabular}{lccc}
\hline & $\begin{array}{c}\text { With } \\
\text { GERD }\end{array}$ & $\begin{array}{c}\text { Without } \\
\text { GERD }\end{array}$ & P value \\
\hline Number & 35 & 5 & $\leq 0.001$ \\
Age (mean) & $41.5 \pm 11.3$ & $44.3 \pm 8.5$ & 0.461 \\
Sex (females) & 25 & 7 & $\leq 0.001$ \\
$\quad$ (males) & 6 & 2 & 0.05 \\
BMI (kg/m2) & $43.3 \pm 3.4$ & $42.1 \pm 2.6$ & 0.342 \\
\hline
\end{tabular}




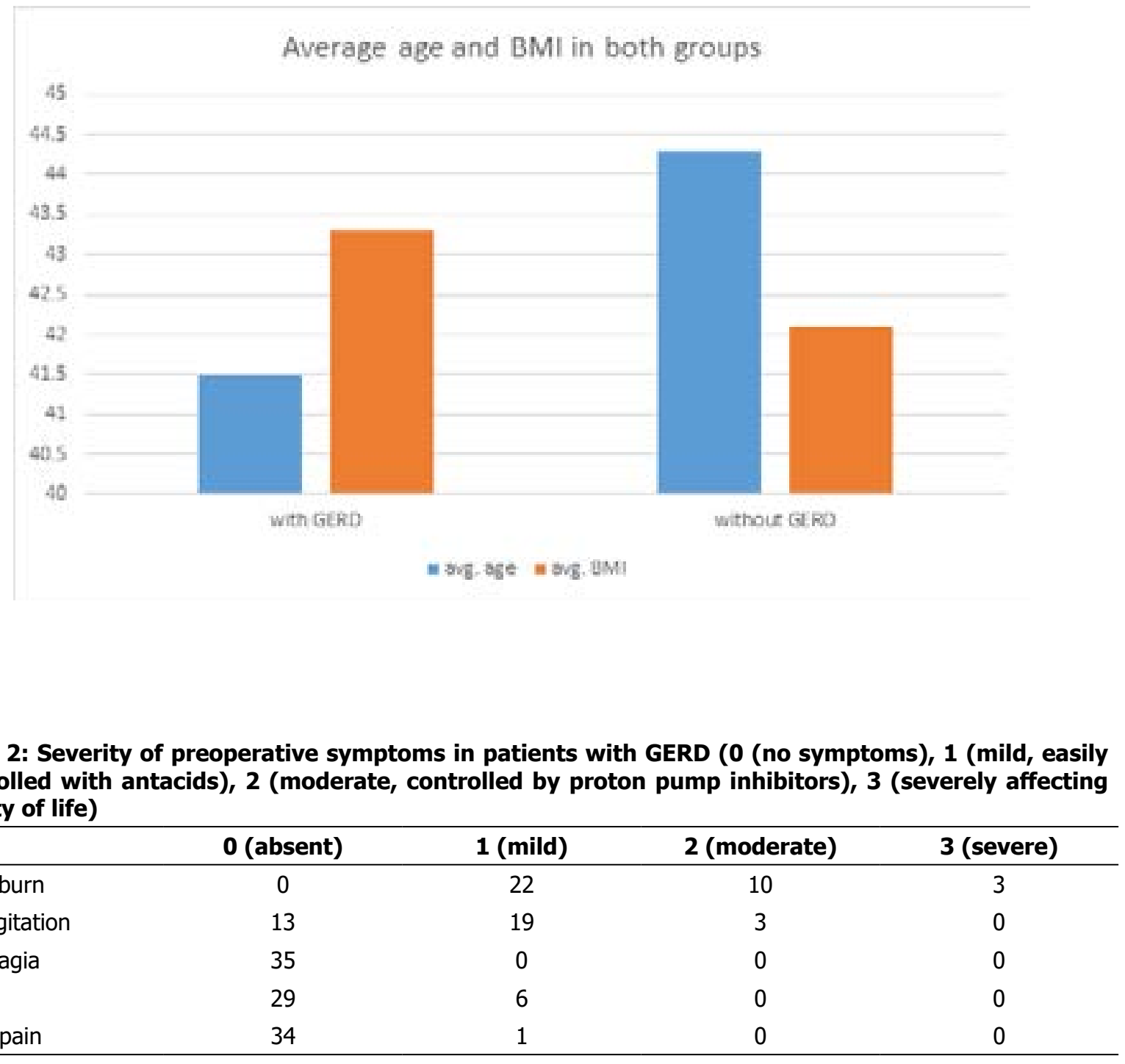

Table 2: Severity of preoperative symptoms in patients with GERD (0 (no symptoms), 1 (mild, easily controlled with antacids), 2 (moderate, controlled by proton pump inhibitors), 3 (severely affecting quality of life)

\begin{tabular}{lcccc}
\hline & 0 (absent) & $\mathbf{1}$ (mild) & 2 (moderate) & 3 (severe) \\
\hline Heart burn & 0 & 22 & 10 & 3 \\
Regurgitation & 13 & 19 & 3 & 0 \\
Dysphagia & 35 & 0 & 0 & 0 \\
Cough & 29 & 6 & 0 & 0 \\
Chest pain & 34 & 1 & 0 & 0 \\
\hline
\end{tabular}

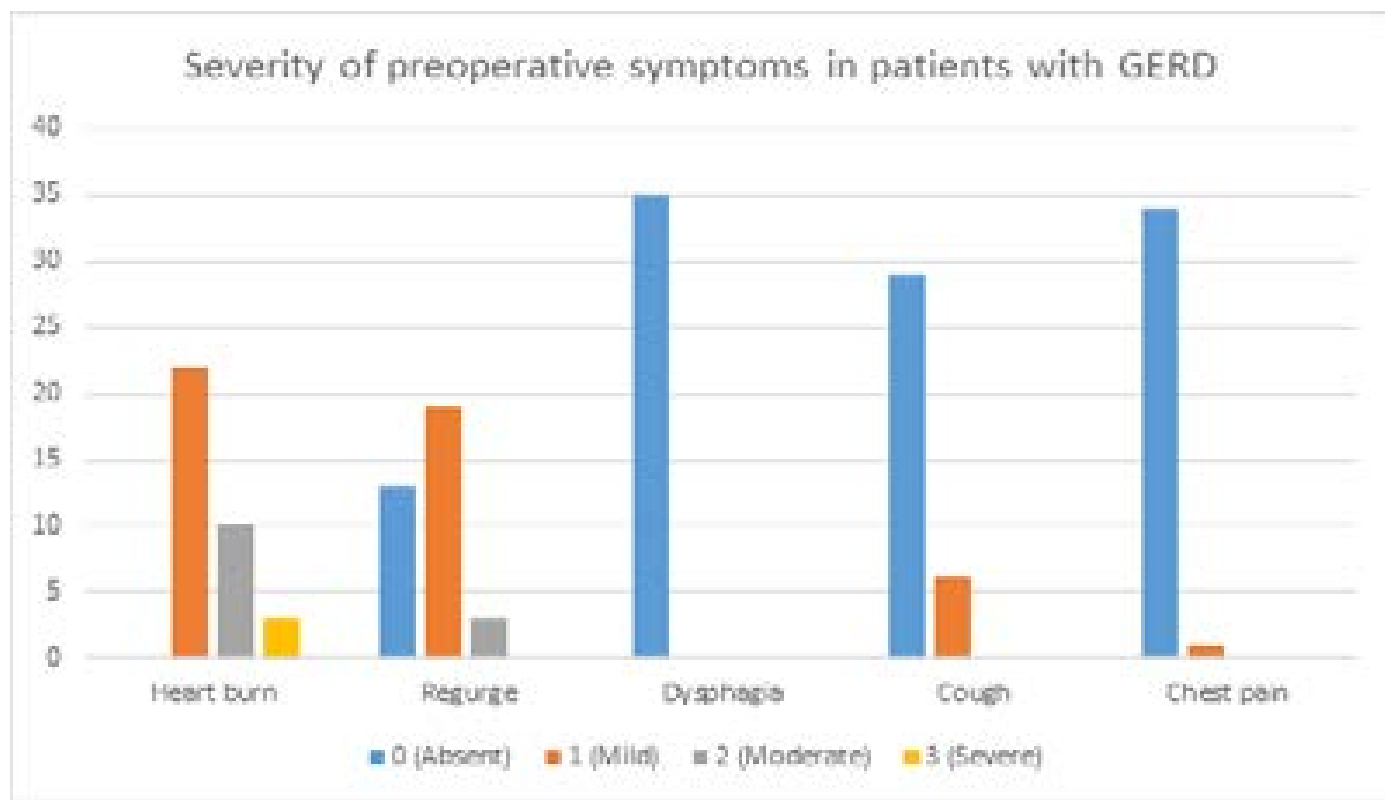


Table 3: Frequency of preoperative symptoms in patients with GERD

\begin{tabular}{lccccc}
\hline & $\mathbf{0}$ (absent) & $\mathbf{1}$ (once/month) & $\mathbf{2}$ (once/week) & $\mathbf{3}$ (2-4/week) & $\mathbf{4}$ (daily) \\
\hline Heart burn & 0 & 6 & 12 & 13 & 2 \\
Regurgitation & 13 & 14 & 5 & 3 & 0 \\
Dysphagia & 35 & 0 & 0 & 0 & 0 \\
Cough & 29 & 4 & 2 & 0 & 0 \\
Chest pain & 34 & 1 & 0 & 0 & 0 \\
\hline
\end{tabular}

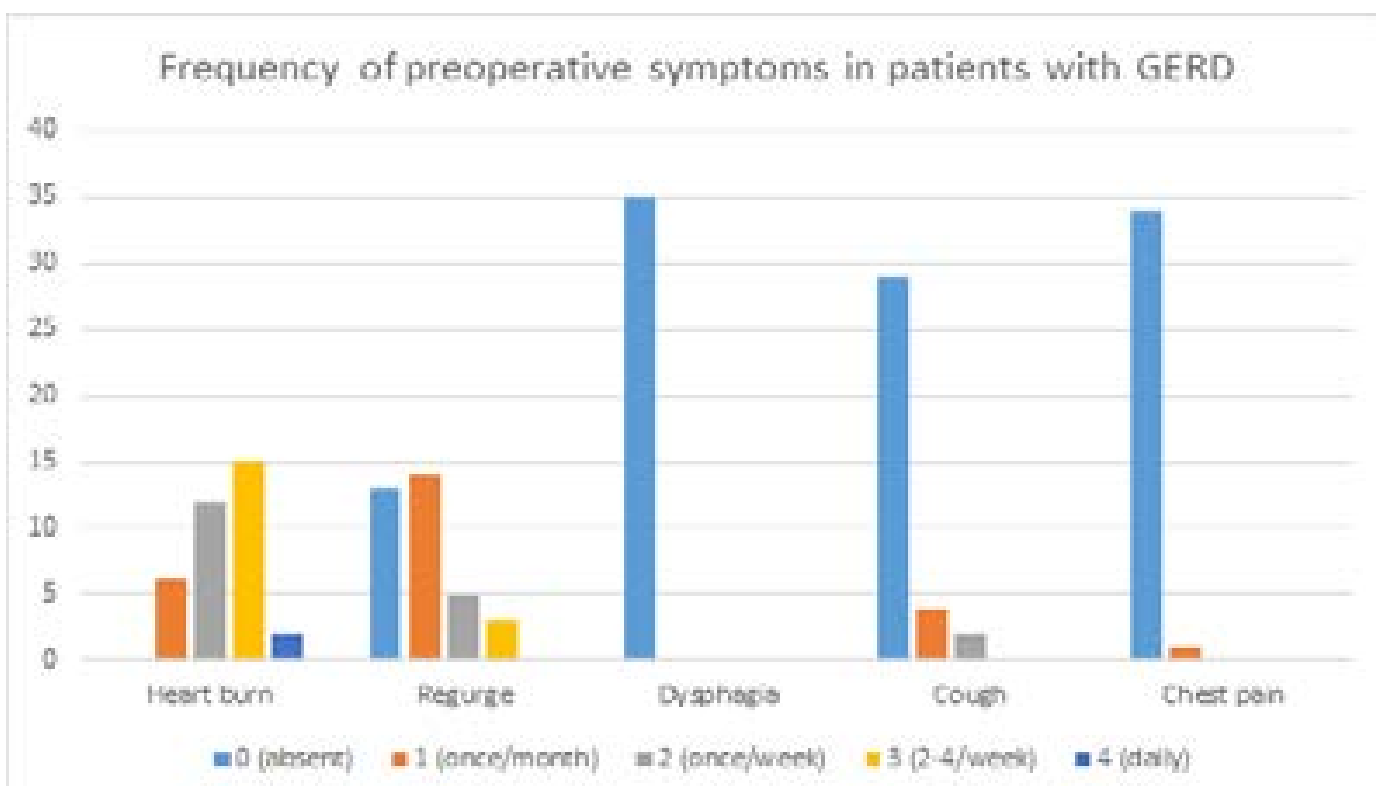

\section{Preoperative Symptomatic assessment:}

Preoperatively, 35 patients had heartburn, 22 had regurgitation, 1 had chest pain, and 6 had cough. None of them experienced dysphagia. Tables 2,3 show scoring of these symptoms as regard severity and frequency.

Barium swallow was only performed preoperatively to exclude patients with short esophagus and hiatal hernia. Upper GIT endoscopy was performed and according to the LA classification, 32 patients had Grade A esophagitis and 3 patient had Grade B esophagitis while 5 patients had no esophagitis.

\section{Postoperative data and follow-up:}

Operative time was $65 \pm 8 \mathrm{~min}$ and length of hospital stay was $48 \mathrm{~h}$. There were no cases of mortality, fistula, or conversion to open surgery. Four patients complained of difficulty tolerating solid food in the early postoperative period. This difficulty resolved completely in three of the four patients after endoscopy alone, and the other patient improved after endoscopic balloon dilatation. The BMI had decreased to $36.5 \pm 5.3 \mathrm{~kg} /$ $\mathrm{m}^{2}$, with $21 \%$ estimated weight loss.

Of the 35 patients with preoperative GERD symptoms, $33(94.3 \%)$ patients continued to experience GERD symptoms with more frequency and increased severity as compared to the pre-operative data recorded (Tables $\mathbf{4 , 5}$ ) but this was statistically insignificant, whereas 2 patients became asymptomatic after surgery.

Table 4: Pre-and post-operative data comparison of the severity of GERD symptoms

\begin{tabular}{lcccccccc}
\hline & \multicolumn{2}{c}{ 0 (absent) } & \multicolumn{2}{c}{$\mathbf{1}$ (mild) } & \multicolumn{2}{c}{ 2 (moderate) } & \multicolumn{2}{c}{ (severe) } \\
\hline & Pre & Post & Pre & Post & Pre & Post & Pre & Post \\
\hline Heart burn & 0 & 2 & 22 & 15 & 10 & 13 & 3 & 5 \\
Regurgitation & 13 & 15 & 19 & 15 & 3 & 4 & 0 & 1 \\
Dysphagia & 35 & 35 & 0 & 0 & 0 & 0 & 0 & 0 \\
Cough & 29 & 31 & 6 & 3 & 0 & 1 & 0 & 0 \\
Chest pain & 34 & 35 & 1 & 0 & 0 & 0 & 0 & 0 \\
\hline
\end{tabular}




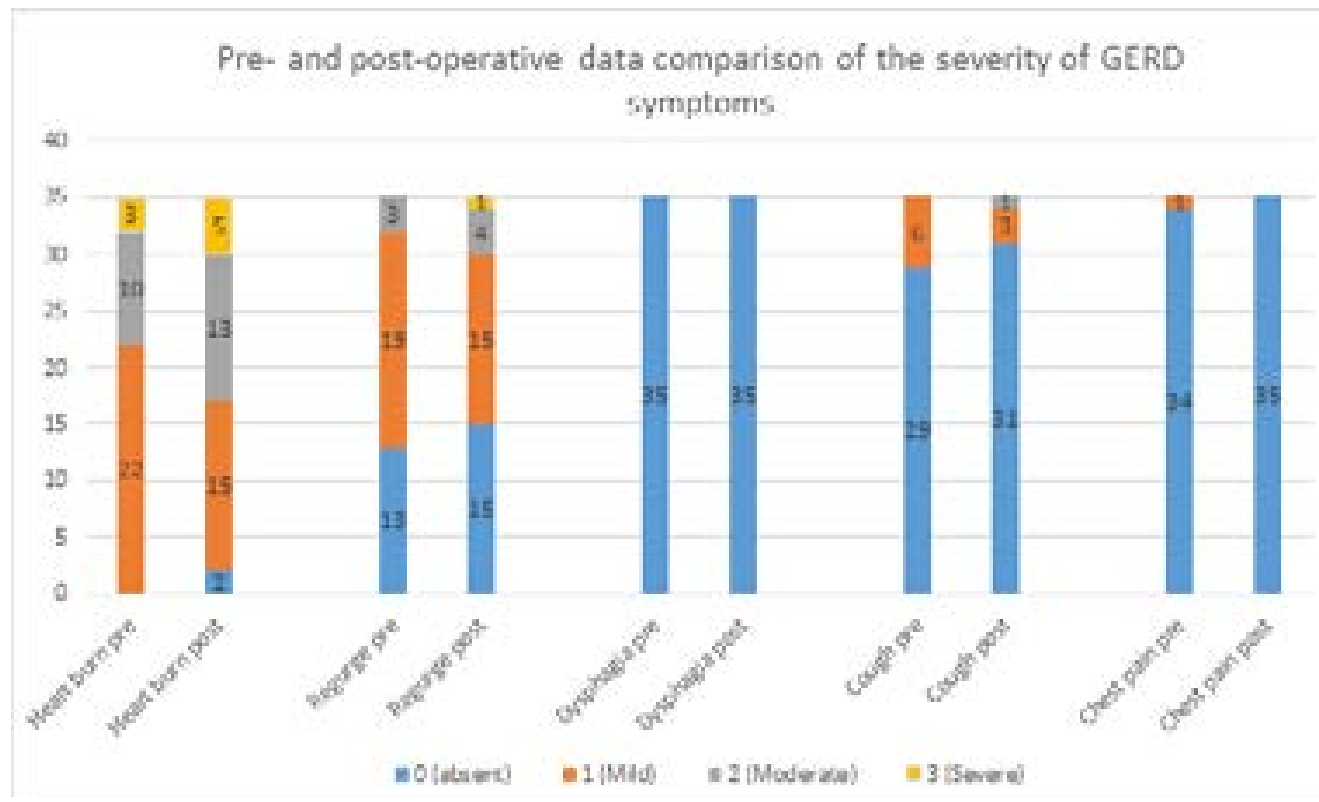

Table 5: Pre-and post-operative data comparison of the severity of GERD symptoms

\begin{tabular}{lcccccccccc}
\hline & \multicolumn{2}{c}{ 0 (absent) } & \multicolumn{2}{c}{ 1 (once/month) } & 2 (once/week) & \multicolumn{3}{c}{ 3 (2-4 /week) } & \multicolumn{2}{c}{ (daily) } \\
\hline Pre & Post & Pre & Post & Pre & Post & Pre & Post & Pre & Post \\
\hline Heart burn & 0 & 2 & 6 & 3 & 12 & 13 & 15 & 16 & 2 & 1 \\
Regurg-itation & 13 & 15 & 14 & 10 & 5 & 5 & 3 & 5 & 0 & 0 \\
Dysph-agia & 35 & 35 & 0 & 0 & 0 & 0 & 0 & 0 & 0 & 0 \\
Cough & 29 & 31 & 4 & 3 & 2 & 1 & 0 & 0 & 0 & 0 \\
Chest pain & 34 & 35 & 1 & 0 & 0 & 0 & 0 & 0 & 0 & 0 \\
\hline
\end{tabular}

Pre- and post-operative data comparison of the frequency of GERD symptoms

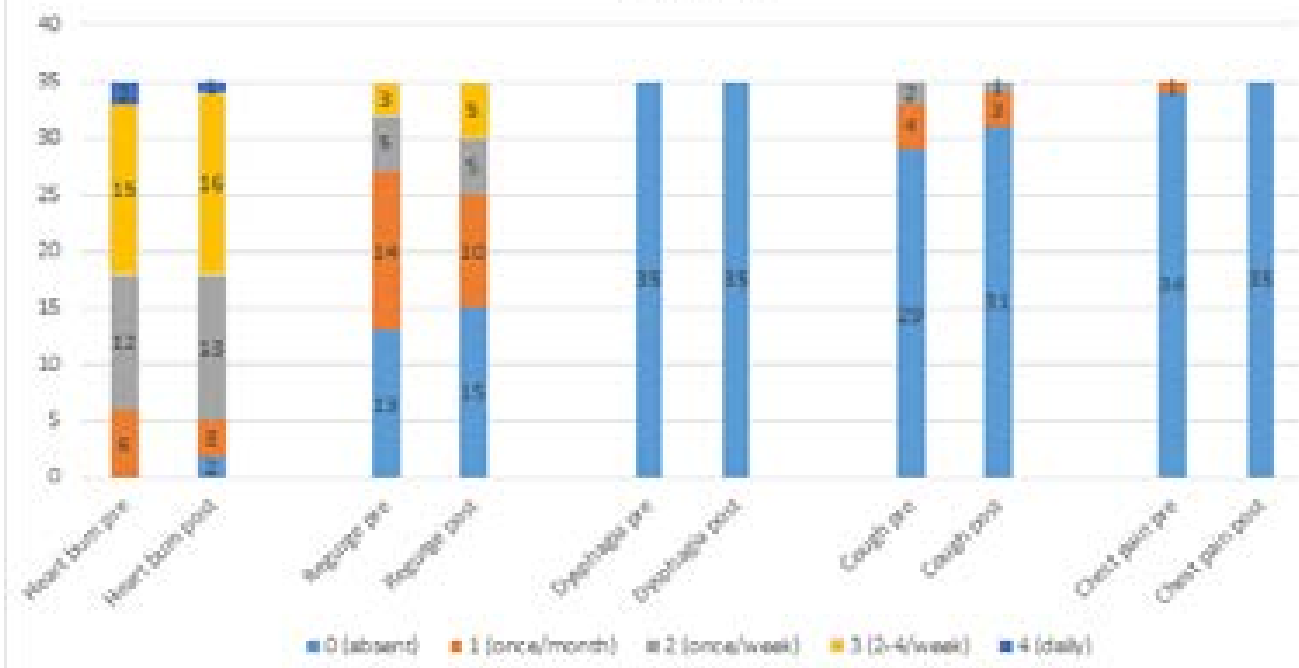


In the subset of 5 patients without GERD symptoms at preoperative evaluation, 1 patient (20\%) developed symptomatic GERD within the 6 months follow-up in the form of heart burn and regurgitation (once/month) which was easily controlled by antacids. Postoperative endoscopy did not show esophagitis.

All patients with pre-operative GERD symptoms received the scheduled follow-up upper GIT endoscopy 6 months post-operatively. That revealed insignificant worsening of esophagitis when compared to preoperative data (29 patients with grade $A$ vs 32 patients pre-operative, $p$ value 0.341 and 6 patients with grade $B$ vs 3 patients pre-operatively, $\mathrm{p}$ value 0.07 ).

\section{Discussion}

There is consensus that bariatric surgery is considered the gold standard treatment for morbid obesity. Nevertheless, the effect of bariatric surgery on GERD needs to be further investigated. Bariatric surgical procedures such as laparoscopic Roux en- $Y$ gastric bypass (RYGB) have been shown to improve symptoms related to GERD. ${ }^{1}$ Laparoscopic sleeve gastrectomy (LSG) has been proven to be an effective weight loss procedure and has gained wide acceptance as stand-alone surgical option for the treatment of morbid obesity, ${ }^{2}$ it is technically easier and is associated with lower postoperative morbidity and mortality rates than RYGB. ${ }^{3}$ However, the effect of LSG on GERD remains controversial, obese patients with GERD may represent a dilemma when choosing the surgical technique: ${ }^{4}$ What would be the effect of LSG on GERD? Is LSG truly associated with GERD? If so, will GERD get better once the patient losses weight or the antireflux barrier will be damaged forever?

Although the underlying mechanisms contributing to the occurrence of GERD after LSG are not clear, the involvement of the lower esophageal sphincter (LES) might play an important role. LSG involves the resection at the angle of His and partial sectioning of the sling fibers, which may impair the function of the LES after LSG. ${ }^{5}$ Those anatomical changes might impair upper stomach emptying into the antrum and could predispose patients to postoperative GERD. Other changes related to GERD after LSG include dilated upper sleeve and narrowing of the midstomach, hypotensive LES, reduced gastric compliance with higher intragastric pressure, decreased gastric emptying, late dilatation of the sleeve, and occurrence of hiatal hernia. ${ }^{6}$ On the contrary, factors associated with reduced GERD after LSG include weight loss, restoration of the angle of His, decreased acid production, and accelerated gastric emptying. ${ }^{7}$
Data in the literature remain controversial, some authors assure that LSG is clearly associated with GERD, conversely, some other authors advocated that GERD could improve or even resolve after LSG.

Both works of Carter et al. ${ }^{8}$ and Howard et al. ${ }^{9}$ were single institution retrospective chart reviews focused on the relationship between GERD and SG. Carter et al. presented 176 patients and recorded weight loss at 6, 12, and 24 months. Data for GERD was presented in two categories: early (symptoms occurring in the first 30 days) and late (symptoms occurring after 30 days). Early post-SG GERD prevalence was increased $14.4 \%$ from the preoperative value, and late post-SG GERD was increased by $12.6 \%$, Howard et al, demonstrated a $14 \%$ increase with a mean follow-up time of 32 weeks, same was the work of Lakdawala et al. ${ }^{10}$ a single institution retrospective study designed to compare SG with RYGB. It provided data that demonstrated a $4 \%$ increase in GERD prevalence after LSG.

In contrast to the above studies, GERD prevalence decreased between $2.8 \%$ and $20 \%$ in the analyzed literature. Melissas et al. ${ }^{11,12}$ demonstrated a decrease in GERD after LSG at its terminal follow-up (24 months) while its 6month analysis demonstrated a $21.7 \%$ increase in prevalence. The works of Chopra et al. ${ }^{13}$ and Rawlins et al. ${ }^{14}$ were both recent retrospective single center studies, which reported decreases in GERD prevalence after LSG of $0.5 \%$ and $4.1 \%$, respectively.

Little information regarding the prevalence of $\mathrm{EE}$ after LSG is available. Braghetto et al. ${ }^{15,16}$ reported that 26 of 167 patients (15.5\%) developed de novo esophagitis within 1 to 2 months after LSG but we found that $20 \%$ of patients who had de novo reflux 6 months after LSG didn't have esophagitis as proved by endoscopic evaluation whereas 3 of 35 patients $(8.5 \%)$ with preoperative esophagitis showed worsened esophagitis following surgery.

Clearly, opinions are contradictory. Based on our early experience, we believe that even if there is no consensus on what the effects of LSG on GERD are, our results showed that this operation provoked de novo reflux in $20 \%$ of patients and worsened it in another $94.3 \%$ even though that change did not show statistical significance. That would be enough reason to avoid indicating LSG to patients who are diagnosed with GERD during the preoperative evaluation, most likely these patients, even if they are in the lower BMI range, would benefit from a gastric bypass.

However, our study had some limitations. A longer 
follow-up period is required to elucidate the serial changes in GERD after LSG. Also, further studies including manometry, $\mathrm{pH}$ measurements, and motility are necessary to elucidate other possible mechanisms contributing to the occurrence of GERD after LSG.

\section{Conclusion}

Although LSG can achieve significant weight loss and improvement of comorbidities in severely obese patients, the prevalence and severity of GERD symptoms and esophagitis increase after the operation, so, we recommend that patients with pre-operative GERD should be offered another bariatric surgery options e.g. gastric bypass.

\section{References}

1. Frezza $\mathrm{EE}$, Ikramuddin $\mathrm{S}$, Gourash $\mathrm{W}$, et al: Symptomaticimprovementingastroesophageal reflux disease (GERD) following laparoscopic Roux-en-Y gastric bypass. Surg Endosc 2002; 16: 1027-1031.

2. Himpens J, Dobbeleir J, Peeters G: Long-term results of laparoscopic sleeve gastrectomy for obesity. Ann Surg 2010; 252: 319-324.

3. Li JF, Lai DD, Lin $\mathrm{ZH}$, et al: Comparison of the long-term results of Rouxen-Y gastric bypass and sleeve gastrectomy for morbid obesity: A systematic review and meta-analysis of randomized and nonrandomized trials. Surg Laparosc Endosc Percutan Tech 2014; 24: 1-11.

4. Burgerhart JS, Schotborgh CA, Schoon EJ, et al: Effect of sleeve gastrectomy on gastroesophageal reflux. Obes Surg 2014; 24: 1436-1441.

5. Weiner RA, Weiner $S$, Pomhoff $I$, et al: Laparoscopic sleeve gastrectomy influence of sleeve size and resected gastric volume. Obes Surg 2007; 17: 1297-1305.

6. Gorodner V, Buxhoeveden R, Clemente G, et al: Does laparoscopic sleeve gastrectomy have any influence on gastroesophageal reflux disease? Preliminary results. Surg Endosc 2015; 29: 1760-1768.
7. Daes J, Jimenez $M E$, SaidN $S$, et al: Laparoscopic sleeve gastrectomy: Symptoms of gastroesophageal reflux can be reduced by changes in surgical technique. Obes Surg 2012; 22: 1874-1879.

8. Carter PR, LeBlanc KA, Hausmann MG, Kleinpeter KP, deBarros SN, Jones SM: Association between gastroesophageal reflux disease and laparoscopic sleeve gastrectomy. Surg Obes Relat Dis 2011; 7: 569-572.

9. Howard DD, Caban AM, Cendan JC, Ben-David K: Gastroesophageal reflux after sleeve gastrectomy in morbidly obese patients. Surg Obes Relat Dis 2011; 7: 709-713.

10. Lakdawala MA, Bhasker A, Mulchandani D, Goel S, Jain S: Comparison between the results of laparoscopic sleeve gastrectomy and laparoscopic Roux-en-Y gastric bypass in the Indian population: A retrospective 1-year study. Obes Surg 2010; 20:1-6.

11. Melissas J, Koukouraki S, Askoxylakis J, et al: Sleeve gastrectomy: A restrictive procedure? Obes Surg 2007; 17: 57-62.

12. Melissas J, Daskalakis M, Koukouraki S, et al: Sleeve gastrectomy: A "food limiting" operation. Obes Surg 2008; 18: 1251-1256.

13. Chopra A, Chao E, Etkin Y, et al: Laparoscopic sleeve gastrectomy for obesity: Can it be considered a definitive procedure? Surg Endosc 2012; 26: 831-837.

14. Rawlins L, Rawlins MP, Brown CC, et al: Sleeve gastrectomy: 5-year outcomes of a single institution. Surg Obes Relat Dis 2013; 9: 21-25.

15. Braghetto I, Lanzarini E, Korn O, et al: Manometric changes of the lower esophageal sphincter after sleeve gastrectomy in obese patients. Obes Surg 2010; 20: 357-362.

16. Braghetto I, Csendes A, Korn O, Valladares $\mathrm{H}$, Gonzalez P, Henriquez A: Gastroesophageal reflux disease after sleeve gastrectomy. Surg Laparosc Endosc Percutan Tech 2010; 20: 148153. 